\title{
The Complexity of Family Medicine Care
}

\author{
Marjorie A. Bowman, MD, MPA
}

In this issue, Katerndahl et $\mathrm{al}^{1}$ quantify the complexity of family physician care compared with cardiology and psychiatry. When accounting for the length of the encounter, the complexity in family medicine is one-third higher than cardiology and 5 times higher than psychiatry.

The measured complexity of family medicine is highly relevant, yet it remains unacknowledged, as evidenced by the reimbursement structure of our health system. If complexity should drive reimbursement, then family physicians should receive increased reimbursement compared with that received by cardiologists or psychiatrists. Alternatively-given that the complexity of family medicine was partially driven by the breath of problems handled by family physicians-perhaps family physicians should be given more time per visit to accomplish the broad range of tasks necessary.

Katerndahl et $\mathrm{al}^{1}$ further note that this complexity per time spent could explain the less successful completion of process measures, such as specific tests or medications, in family medicine. However, I would note that the less successful completion of process measures frequently occurs simultaneously with similar or better outcomes at a group or population level, ${ }^{2}$ such as mortality rates. Family physicians contribute improved outcomes at both the individual and group levels. Process measures do not necessarily reflect important outcomes; yes, process is easier to measure, but sometimes it is lacking sufficient substance.

The complexity of the interplay of multiple facets of human health creates at least some of the common disconnect between process measures and patient outcomes. These expected process measures have been shown by some research studies to

This article was not externally peer reviewed.

Conflict of interest: The author is an editor of JABFM.

See Related Article on Page 6. relate to outcomes, but often have been applied to a single disease with limited variation in patient characteristics, comorbidities, and measured outcomes and in a circumstance in which the health care is covered by insurance or the study itself. Thus, for many disease states and patients with multiple diseases, these process measures add little, and sometimes no, benefit. Drug $\mathrm{X}$ is compared with drug $\mathrm{Y}$ for disease $\mathrm{A}$ in patients with diseases $\mathrm{K}, \mathrm{L}$, and $\mathrm{M}$; drug $\mathrm{X}$ is associated with a $5 \%$ lower hospitalization rate during the next year. However, family medicine patients have diseases $\mathrm{K}, \mathrm{L}, \mathrm{M}, \mathrm{N}$, $\mathrm{O}, \mathrm{P}$, and Q; less expensive drug Y may help 2 of these problems at once to make a $10 \%$ difference in quality of life and improve the patient's ability to care for a family member; thus, family physicians will order drug Y, but get "dinged" for not ordering drug $\mathrm{X}$. Or, as another example, how important is it to check urine microalbumin levels once a year in a patient with diabetes when the family physician is helping the patient work through depression related to a parent's death, increased tobacco use, and a glycosylated hemoglobin level of $10 \%$ because the patient can't afford the copay?

Another source of the disconnect between process and outcomes is that it is the first action taken, such as the first drug added or the first preventive test completed, ${ }^{3}$ which provides most of the gain in outcome, meaning that additional processes provide little additional benefit. Thus, when time is short and many different complaints must be addressed, implying that many process measures for each complaint or disease could be expected, family physicians often do the next process measure that could make the most difference for that patient. Family physicians prioritize patient outcome over meeting externally defined process measures.

Of course, many physicians and medical students have already figured out that family physician care is more complex and that the breadth of problems is a key driver of this. It basically encapsulates why some say that family medicine is too difficult-one has to know too much and do too many 
types of things (breadth), and/or family medicine is too easy-one does not have to know unusual or rare conditions (depth). After all, it is mostly well care for patients of all ages, hypertension, diabetes, acute nonemergency care, asthma, depression, heart failure, obesity, pain, drug or physical abuse, the list keeps going on and on. It can seem so "anti-process measure" to consider that taking care of several diseases at the same time can create better outcomes than taking care of each separately.

It is this complexity of the human condition and the interplay of these many factors in people's lives that creates the complexity of family practice, and this is actually what draws us to family medicine, the integrator and scaffold for people's health. Family medicine: complex even in its complexity, and powerful in making a difference.

\section{References}

1. Katerndahl D, Wood R, Jaén CR. Family medicine outpatient encounters are more complex than those of cardiology and psychiatry. J Am Board Fam Med 2011;24:6-15.

2. Stange KC, Ferrer RL. The paradox of primary care. Ann Fam Med 2009;7:293-9.

3. Mold JW, Hamm RM, McCarthy LH. The law of diminishing returns in clinical medicine: how much risk reduction is enough? J Am Board Fam Med 2010;23:371-5. 